Case Report

\title{
Evading Capture by Residual Disease Monitoring: Extramedullary Manifestation of JAK2 V617F-Positive Primary Myelofibrosis After Allogeneic Stem Cell Transplantation
}

\author{
Stephen E. Langabeer, ${ }^{1}$ James Nolan, ${ }^{2}$ Karl Haslam, ${ }^{1}$ Lindsey Clarke, \\ Richard Flavin, ${ }^{3}$ and Eibhlin Conneally ${ }^{2}$ \\ ${ }^{1}$ Cancer Molecular Diagnostics, St. James's Hospital, Dublin 8, Ireland \\ ${ }^{2}$ Department of Haematology, St. James's Hospital, Dublin 8, Ireland \\ ${ }^{3}$ Department of Histopathology, St. James's Hospital, Dublin 8, Ireland \\ Correspondence should be addressed to Stephen E. Langabeer; slangabeer@stjames.ie
}

Received 15 June 2015; Revised 17 July 2015; Accepted 22 July 2015

Academic Editor: Ramon Tiu

Copyright ( 2015 Stephen E. Langabeer et al. This is an open access article distributed under the Creative Commons Attribution License, which permits unrestricted use, distribution, and reproduction in any medium, provided the original work is properly cited.

\begin{abstract}
Monitoring of the JAK2 V617F allele burden in myeloproliferative neoplasms after allogeneic stem cell transplantation is useful to determine levels of residual disease and has the potential to detect early relapse and guide subsequent clinical intervention. A case is described of a JAK2 V617F-positive primary myelofibrosis patient who underwent allogeneic stem cell transplantation. Prospective residual disease monitoring of the peripheral blood failed to detect an extramedullary manifestation of the disease, a periorbital myeloid sarcoma, arising nearly three years after transplant. This case serves to highlight a pitfall in residual disease monitoring for myeloproliferative neoplasm-associated mutations in the post-allogeneic stem cell transplantation setting.
\end{abstract}

\section{Introduction}

Primary myelofibrosis (PMF) is one of the classical Philadelphia chromosome-negative myeloproliferative neoplasms and is clinically characterized by cytopenias, splenomegaly, and multiple disease-related symptoms that reduce the quality of life. The clinical course of PMF is variable with an increased risk of progression to acute myeloid leukemia (AML) as compared to essential thrombocythemia and polycythemia vera [1]. While the introduction of JAK2 inhibitors has improved the outlook for many patients with PMF [2], the only curative option that remains is allogeneic stem cell transplantation (ASCT). Improvements in candidate patient selection and stratification, timing of transplantation, and conditioning regimens have significantly reduced the transplant related morbidity and increased the overall survival for patients undergoing this procedure [3]. The most commonly observed acquired mutations in PMF are the JAK2 V617F, $C A L R$ exon 9 mutations, and $M P L$ exon 10 mutations with $J A K 2$ V617F and CALR mutation status indicative of overall survival after ASCT $[4,5]$. Tracking disease burden utilising these patient-specific mutations provides an individualized approach allowing assessment of disease clearance and providing a marker with which to guide adoptive immunotherapy [6-9].

A case is described in which JAK2 V617F residual disease monitoring failed to detect an extramedullary manifestation of PMF in a patient after ASCT and which serves to highlight a pitfall of this approach.

\section{Case Report}

Whilst undergoing imaging of a knee replacement for osteoarthritis three years previously, a 54-year-old male was noted to have patchy skeletal sclerosis. Further investigations revealed splenomegaly with a nine-month history of progressive constitutional symptoms (generalised unwellness), lethargy, night sweats, and weight loss. Initial hematological investigation revealed a hemoglobin of $10.3 \mathrm{~g} / \mathrm{dL}$, a white cell count of $7.8 \times 10^{9} / \mathrm{L}$, platelets of $53 \times 10^{9} / \mathrm{L}$, and elevated 


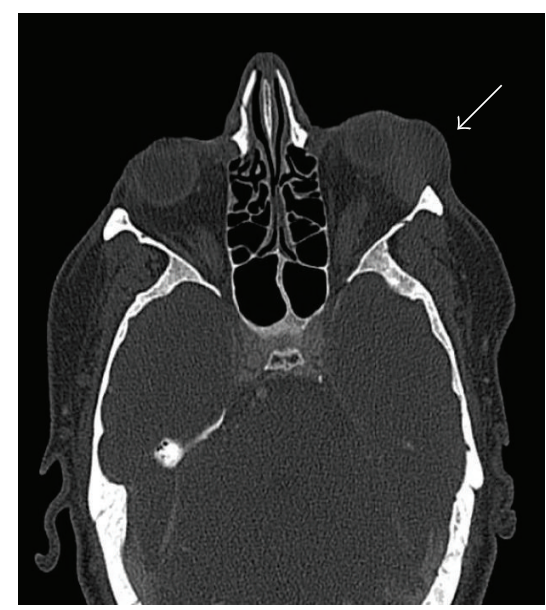

(a)

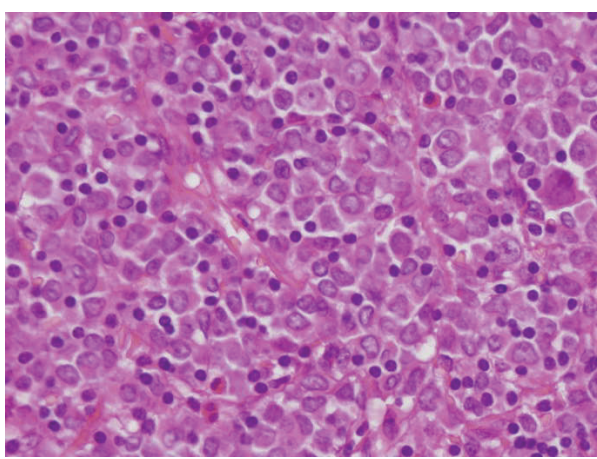

(b)

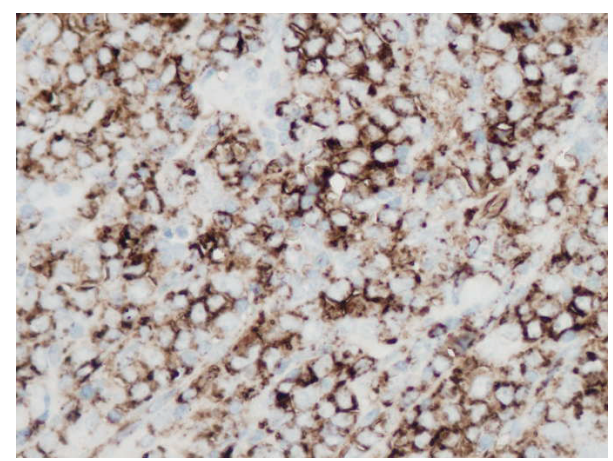

(c)

FIGURE 1: (a) CT showing left periorbital myeloid sarcoma (arrowed); (b) periorbital biopsy showing blast infiltration by myeloid sarcoma (Hematoxylin and Eosin; magnification $\times 40$ ); (c) positive immunohistochemical staining for CD34 in the myeloid sarcoma (magnification $\times 40)$.

reticulocyte count $\left(212 \times 10^{9} / \mathrm{L}\right)$ and lactate dehydrogenase (LDH; 990 IU/L). The blood film was leukoerythroblastic with red cell anisopoikilocytosis, tear drop cells, basophilic stippling, and $2 \%$ circulating blasts. Bone marrow aspirate was unsuccessful; however the bone marrow biopsy showed abnormal marrow architecture and grade IV reticulin fibrosis. The JAK2 V617F mutation was detected by qualitative PCR confirming the diagnosis of PMF. Four months later the platelet count had fallen to $24 \times 10^{9} / \mathrm{L}$ with $3 \%$ blast cells in the peripheral blood. The LDH was markedly elevated at 1912 IU/L with the patient now noted to have a splenomegaly $12 \mathrm{~cm}$ below the costal margin. His risk status based on International Prognostic Scoring System was intermediate-2 [10].

The patient proceeded to reduced intensity conditioning ASCT [11] with a matched sibling donor. The posttransplant course was complicated by Klebsiella bacteremia and grade II skin graft-versus-host disease (GVHD). With the introduction of corticosteroids the patient developed Cytomegalovirus reactivation requiring readmission, steroid-induced hypomania, and steroid-induced insulin-dependent diabetes mellitus. A robust and validated quantitative PCR (qPCR) assay for JAK2 V617F [12-14] was employed to determine the allele burden before ASCT (30.7\%). At two months after ASCT donor chimerism (DC) was 97\% and JAK2 V617F
$0.46 \%$ and at six months after ASCT DC was $96 \%$ and JAK2 V617F 0.79\%. Full DC (100\%) and undetectable JAK2 V61F were concurrently noted at eleven months after ASCT and remained so at prospective three monthly assessments.

In the long-term follow-up clinic at 32 months after ASCT, a $1 \mathrm{~cm}$ hard lump was noted beneath the left eye. Subsequent imaging noted a mass lesion arising within the inferolateral aspect of the left orbit, displacing the globe anteromedially and which appeared to lie within the extraconal space (Figure 1(a)). Excision of the periorbital bulk mass was undertaken with histopathological examination revealing adipose/connective tissue infiltrated by blasts (Figure 1(b)) which stained positively for CD34, CD117, and CD45 (Figure 1(c)) and negatively for CD20, TdT, Granzyme $\mathrm{B}, \mathrm{CD} 68$, and CD1a. The blasts were also JAK2 V617F-positive consistent with a diagnosis of myeloid sarcoma, an extramedullary tumor (EMT). At this time the patient had a hemoglobin level of $14.0 \mathrm{~g} / \mathrm{dL}$, white cell count of $4.2 \times 10^{9} / \mathrm{L}$, neutrophils of $2.0 \times 10^{9} / \mathrm{L}$, and an unchanged, maintained platelet count of $115 \times 10^{9} / \mathrm{L}$. The JAK2 V617F was undetectable in both the peripheral blood and the bone marrow aspirate, the latter showing no morphological evidence of PMF.

The patient was not a candidate for further intensive chemotherapy and underwent involved field radiotherapy to 
the left orbit (30 Gy in 15 fractions) and at last follow-up 41 months after ASCT, the JAK2 V617F remained undetectable in the peripheral blood.

\section{Discussion}

Despite the fact that ASCT remains the only curative option for PMF, the five-year overall survival with reduced intensity conditioning is approximately $50 \%$, with relapse being the major cause of treatment failure which is often associated with an aggressive clinical course, primarily due to clonal evolution [15]. Close molecular monitoring after ASCT is employed to determine the rate and status of disease clearance and to predict impending relapse allowing prompt clinical intervention. In those PMF patients in whom sensitive, residual disease monitoring of the JAK2 V617F allele burden has been performed, reemergence of this clone in either the peripheral blood or bone marrow precedes loss of DC and clinical relapse $[16,17]$. A potential confounding factor of this qPCR approach is the possibility of clonal evolution in which the JAK2 V617F relinquishes its role as the main driving mutation of this disease. While early detection of extramedullary relapse of AML cases using residual disease monitoring of AML-associated genetic markers has been documented, evidence for a similar benefit in JAK2 V617Fpositive PMF, particularly after ASCT, is scarce [18-20].

EMT arising after ASCT for hematological malignancies appears to be predominantly associated with an initial diagnosis of AML but has been noted in association with other myeloid and lymphoid malignancies and they may occur in the skin and soft tissues, central nervous system, and gastrointestinal tract and at other sites [21]. EMTs are due to the migration of abnormal neoplastic hematopoietic precursor cells into the tissues and therefore formation should be considered a metastatic process [22, 23]. EMTs occurring after ASCT may possess different clinical characteristics compared to bone marrow relapses such as a longer duration of onset and an association with GVHD, both apparent in the case described herein [21]. The mechanism(s) by which extramedullary disease relapse remains molecularly undetected in the peripheral blood remains unknown: one possibility is evasion of PMF stem cells from immunological detection by residing in a shielded niche.

Residual disease monitoring using JAK2 V617F qPCR in PMF after ASCT is effective in predicting relapse in the majority of those patients in whom the disease is driven by this mutation. However, vigilance is required as extramedullary manifestations of PMF, although rare, may evade early capture by this approach.

\section{Conflict of Interests}

The authors declare that there is no conflict of interests regarding the publication of this paper.

\section{References}

[1] A. Tefferi, "Primary myelofibrosis: 2014 update on diagnosis, risk-stratification, and management," American Journal of Hematology, vol. 89, no. 9, pp. 915-925, 2014.
[2] A. Rosenthal and R. A. Mesa, "Janus kinase inhibitors for the treatment of myeloproliferative neoplasms," Expert Opinion on Pharmacotherapy, vol. 15, no. 9, pp. 1265-1276, 2014.

[3] D. Babushok and E. Hexner, "Allogeneic transplantation for myelofibrosis: for whom, when, and what are the true benefits?" Current Opinion in Hematology, vol. 21, no. 2, pp. 114-122, 2014.

[4] H. Alchalby, A. Badbaran, T. Zabelina et al., "Impact of JAK2V617F mutation status, allele burden, and clearance after allogeneic stem cell transplantation for myelofibrosis," Blood, vol. 116, no. 18, pp. 3572-3581, 2010.

[5] V. Panagiota, F. Thol, B. Markus et al., "Prognostic effect of calreticulin mutations in patients with myelofibrosis after allogeneic hematopoietic stem cell transplantation," Leukemia, vol. 28, no. 7, pp. 1552-1555, 2014.

[6] N. Kröger, H. Alchalby, E. Klyuchnikov et al., "JAK2-V617F triggered preemptive and salvage adoptive immunotherapy with donor-lymphocyte infusion in patients with myelofibrosis after allogeneic stem cell transplantation," Blood, vol. 113, no. 8, pp. 1866-1868, 2009.

[7] T. Lange, A. Edelmann, U. Siebolts et al., "JAK2 p.V617F allele burden in myelo proliferative neoplasms one month after allogeneic stem cell transplantation significantly predicts outcome and risk of relapse," Haematologica, vol. 98, no. 5, pp. 722-728, 2013.

[8] K. Haslam, S. E. Langabeer, K. Molloy, M. F. McMullin, and E. Conneally, "Assessment of CALR mutations in myelofibrosis patients, post-allogeneic stem cell transplantation," British Journal of Haematology, vol. 166, no. 5, pp. 800-802, 2014.

[9] H. Alchalby, A. Badbaran, O. Bock et al., "Screening and monitoring of MPL W515L mutation with real-time PCR in patients with myelofibrosis undergoing allogeneic-SCT," Bone Marrow Transplantation, vol. 45, no. 9, pp. 1404-1407, 2010.

[10] F. Cervantes, B. Dupriez, A. Pereira et al., "New prognostic scoring system for primary myelofibrosis based on a study of the International working group for myelofibrosis research and treatment," Blood, vol. 113, no. 13, pp. 2895-2901, 2009.

[11] N. Kröger, E. Holler, G. Kobbe et al., "Allogeneic stem cell transplantation after reduced-intensity conditioning in patients with myelofibrosis: a prospective, multicenter study of the Chronic Leukemia Working Party of the European Group for Blood and Marrow Transplantation," Blood, vol. 114, no. 26, pp. 5264-5270, 2009.

[12] T. S. Larsen, J. H. Christensen, H. C. Hasselbalch, and N. Pallisgaard, "The JAK2 V617F mutation involves B- and T-lymphocyte lineages in a subgroup of patients with Philadelphiachromosome negative chronic myeloproliferative disorders," British Journal of Haematology, vol. 136, no. 5, pp. 745-751, 2007.

[13] J. V. Jovanovic, A. Ivey, A. M. Vannucchi et al., "Establishing optimal quantitative-polymerase chain reaction assays for routine diagnosis and tracking of minimal residual disease in JAK2-V617F-associated myeloproliferative neoplasms: a joint European LeukemiaNet/MPN\&MPNr-EuroNet (COST action BM0902) study," Leukemia, vol. 27, no. 10, pp. 2032-2039, 2013.

[14] K. Haslam, K. M. Molloy, E. Conneally, and S. E. Langabeer, "Evaluation of a JAK2 V617F quantitative PCR to monitor residual disease post-allogeneic hematopoietic stem cell transplantation for myeloproliferative neoplasms," Clinical Chemistry and Laboratory Medicine, vol. 52, no. 3, pp. e29-e31, 2014.

[15] V. Gupta, A. K. Malone, P. N. Hari et al., "Reduced-intensity hematopoietic cell transplantation for patients with primary 
myelofibrosis: a cohort analysis from the Center for International Blood and Marrow Transplant Research," Biology of Blood and Marrow Transplantation, vol. 20, no. 1, pp. 89-97, 2014.

[16] N. K. Steckel, M. Koldehoff, M. Ditschkowski, D. W. Beelen, and A. H. Elmaagacli, "Use of the activating gene mutation of the tyrosine kinase (VAL617Phe) JAK2 as a minimal residual disease marker in patients with myelofibrosis and myeloid metaplasia after allogeneic stem cell transplantation," Transplantation, vol. 83, no. 11, pp. 1518-1520, 2007.

[17] N. Kröger, A. Badbaran, E. Holler et al., "Monitoring of the JAK2-V617F mutation by highly sensitive quantitative real-time PCR after allogeneic stem cell transplantation in patients with myelofibrosis," Blood, vol. 109, no. 3, pp. 1316-1321, 2007.

[18] S. Yoshihara, H. Tamaki, K. Ikegame et al., "Early prediction of extramedullary relapse of leukemia following allogeneic stem cell transplantation using the WT1 transcript assay," Biology of Blood and Marrow Transplantation, vol. 12, no. 2, p. 86, 2006.

[19] H. Tamaki, S. Yoshihara, T. Fujioka, M. Kawakami, Y. Oka, and H. Ogawa, "Molecular detection of AML1-MTG8-positive cells in peripheral blood from a patient with isolated extramedullary relapse of $\mathrm{t}(8 ; 21)$ acute myeloid leukemia," Leukemia, vol. 23, no. 2, pp. 424-426, 2009.

[20] W. Y. Au, A. Fung, A. K. Lie, K. Y. Lam, C. C. Lam, and Y. L. Kwong, "Reemergence of JAK2 V617F clone heralds extramedullary leukemia relapse after BMT for transformed essential thrombocytosis," Annals of Hematology, vol. 86, no. 2, pp. 145147, 2007.

[21] Q. Huang, D. Reddi, P. Chu, D. S. Snyder, and D. D. Weisenburger, "Clinical and pathologic analysis of extramedullary tumors after hematopoietic stem cell transplantation," Human Pathology, vol. 45, no. 12, pp. 2404-2410, 2014.

[22] M. A. Haniffa, B. S. Wilkins, C. Blasdale, and N. B. Simpson, "Cutaneous extramedullary hemopoiesis in chronic myeloproliferative and myelodysplastic disorders," Journal of the American Academy of Dermatology, vol. 55, supplement 2, pp. S28-S31, 2006.

[23] G. R. Fraga and S. K. Caughron, "Cutaneous myelofibrosis with JAK2 V617F mutation: metastasis, not merely extramedullary hematopoiesis!," The American Journal of Dermatopathology, vol. 32, no. 7, pp. 727-730, 2010. 


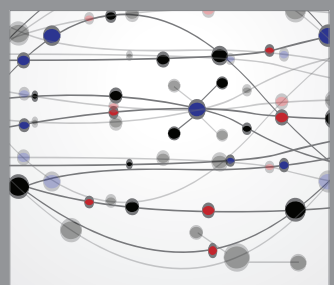

The Scientific World Journal
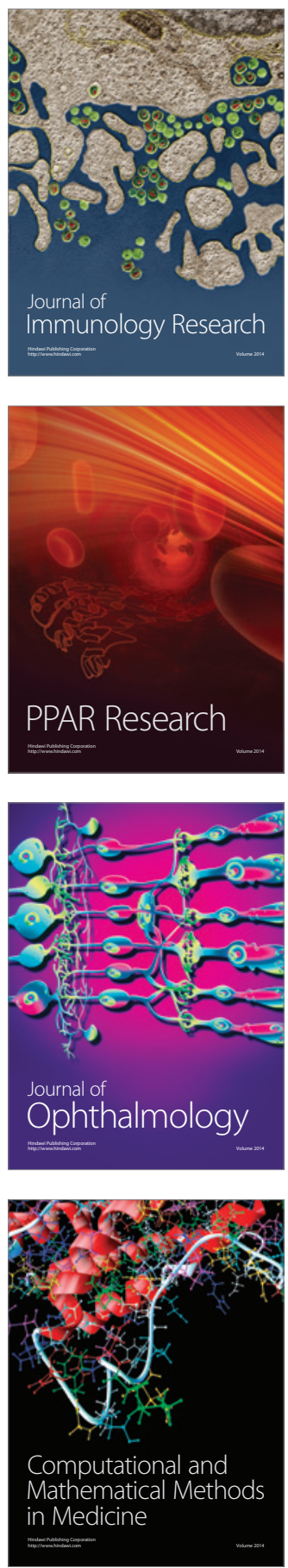

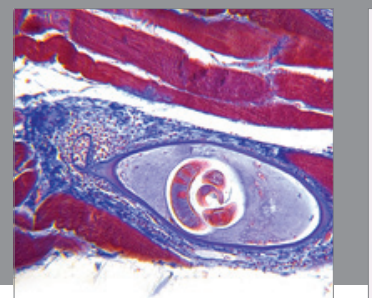

Gastroenterology

Research and Practice
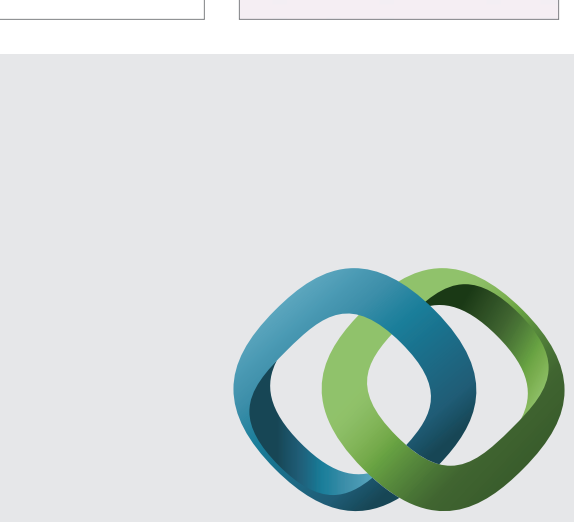

\section{Hindawi}

Submit your manuscripts at

http://www.hindawi.com
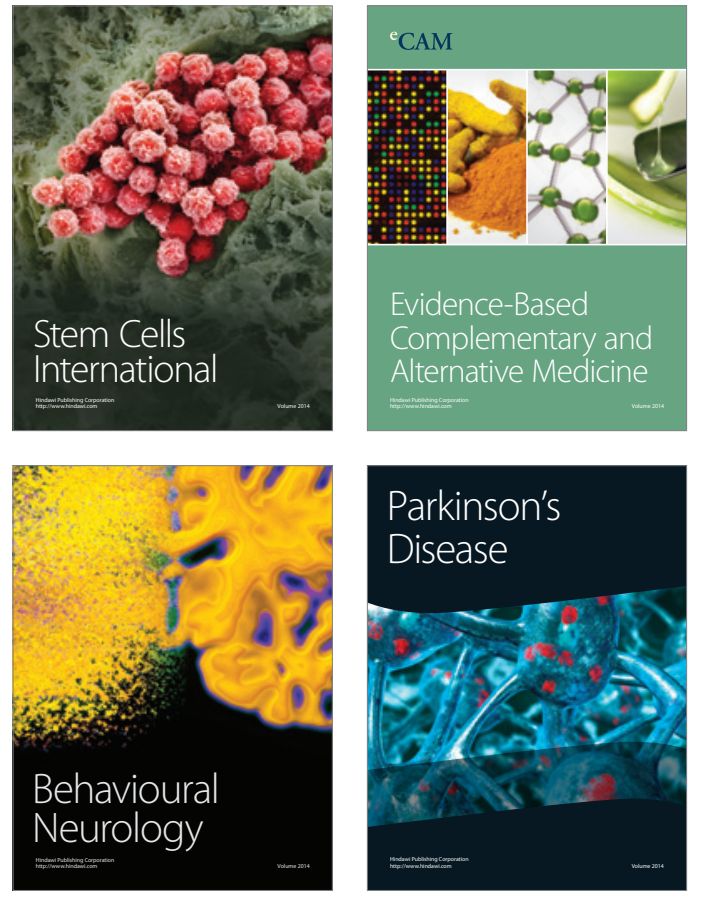
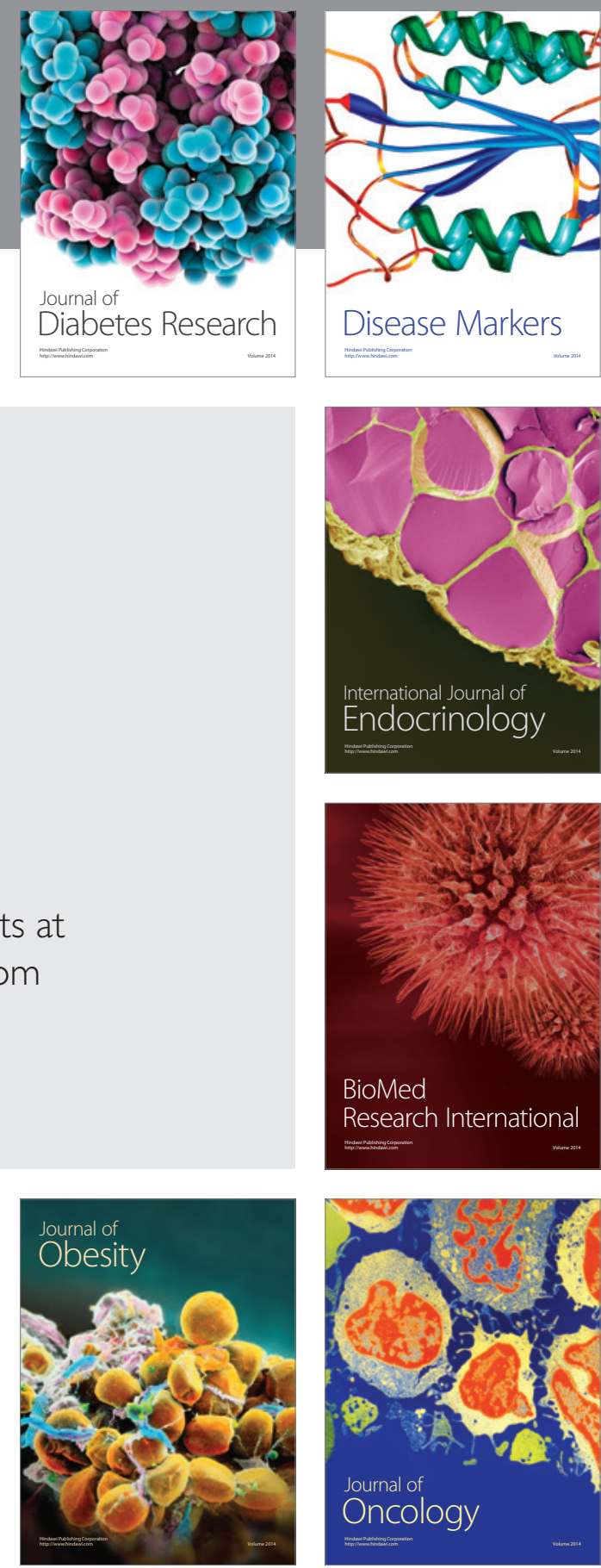

Disease Markers
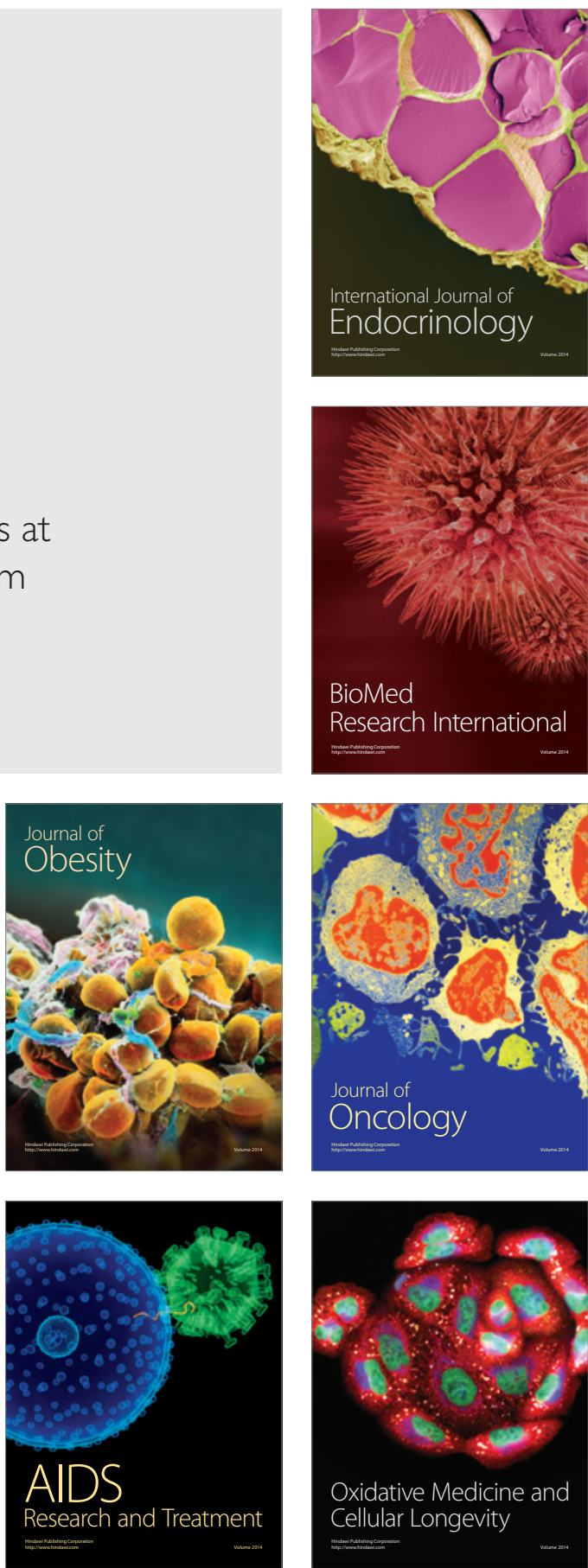